\title{
A Sinister View of Dilation Equations
}

\author{
David Malone*
}

21 December 2001

\begin{abstract}
We present a technique for studying refinable functions which are compactly supported. Refinable functions satisfy dilation equations and this technique focuses on the implications of the dilation equation at the edges of the support of the refinable function. This method is fruitful, producing results regarding existence, uniqueness, smoothness and rate of growth of refinable functions.
\end{abstract}

\section{Introduction}

An $m$-refinable function $f$ is a function which satisfies a dilation equation of scale $m$,

$$
f(x)=\sum_{k \in \mathbb{Z}} c_{k} f(m x-k)
$$

Dilation equations, refinable functions and their properties are often of interest to those working on wavelet bases, curve and surface interpolation in computer graphics, self-similarity and related fields.

A case of particular interest has been the case where $f$ is compactly supported. This case has been studied using both Fourier Analysis and analysis of the linear operators given by evaluating the right hand side of the dilation equation. These analyses provide insight into the existence, uniqueness and smoothness of refinable functions.

In this paper we outline a more direct analysis. If a refinable function is compactly supported then its support must have a left-hand end. In Section 2 we inspect the dilation equation at this end and derive the basic tools (Figure 1 and Equation 2) with which our later results are built.

We proceed by looking at the interaction of these tools with other properties. In Section 3 we give a characterisation of the refinable functions that are constant on the intervals $[n, n+1)$. This class of functions generalises of one of the best known refinable functions, $\chi_{[0,1)}(x)=\chi_{[0,1)}(2 x)+$ $\chi_{[0,1)}(2 x-1)$. In Section 4 we study a different generalisation: finding all 2-refinable characteristic functions. We conjecture that these are actually unions of intervals $[n, n+1)$.

Section 5 gives quick bounds on the smoothness and growth of refinable functions in terms of their coefficients. Consequently we show that there are no $C^{\infty}$ compactly-supported refinable functions.

The classification of functions which are refinable on two scales has proven curiously resistant to study using Fourier Analysis. In Section 6 we show that compactly-supported functions which are 2- and 3-refinable are of the form $\sum a_{l}(x-l)_{+}^{n}$. This result was proved independently in [1] but is a good example of this technique.

This technique is most obviously suited to functions on $\mathbb{R}$, where the meaning of left-hand end is obvious. We study dilation matrices that admit parallelepipeds as self-affine tiles in Section 7 , as an example of how this technique can be applied in $\mathbb{R}^{d}$.

For simplicity we state our results for scale $m=2$ except where other scales are central to or illuminate the result. There are natural extensions of these results to any integer scale.

*Communications Networks Research Institute, Dublin Institute of Technology, Ireland, <David.Malone@dit.ie> 


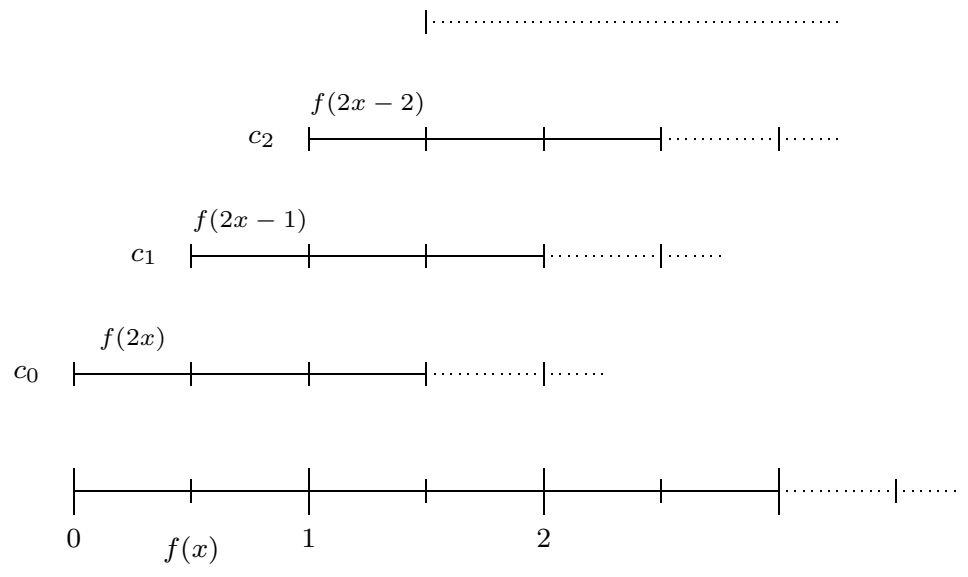

Figure 1: The left-hand end of a dilation equation.

\section{The left-hand end}

We begin by locating the left-hand end of our function. To make life easy we align the left hand end with $x=0$, using the following lemmas. The proof of Lemma 2.1 is straightforward. To prove Lemma 2.2 look at the smallest interval where $f$ is not zero.

Lemma 2.1. Suppose $g(x)=\sum d_{k} g(2 x-k)$, and only finitely many of the $d_{k}$ are non-zero. Then we can find $l$ so that when we translate by $l$ to get $f(x):=g(x-l)$ we have $f(x)=\sum c_{k} f(2 x-k)$, $c_{0} \neq 0$, and $c_{k}=0$ when $k<0$ and $c_{k}=d_{k-l}$.

Lemma 2.2. If $f$ is compactly-supported and satisfies a dilation equation $f(x)=\sum c_{k} f(2 x-k)$, where $c_{0} \neq 0$ and $c_{k}=0$ when $k<0$, then $f$ is zero (almost) everywhere in $(-\infty, 0)$.

Once we have $f$ lined up, we can draw Figure 1 using the fact that $f$ has no support to the left of $x=0$. At the bottom we show the domain of $f$ and above it are the dilates of $f$ which contribute to the dilation equation for a particular value of $x$.

Using Figure 1 we may determine the values of $f$ on all of $\mathbb{R}^{+}$using the values of $f$ on $[0,1 / 2)$. Examining $[0,1 / 2)$ we see that $f(x)=c_{0} f(2 x)$ and substituting $x / 2$ for $x$ we get $f(x)=$ $f(x / 2) / c_{0}$ for $x \in[0,1)$. Thus knowing $f$ on $\left[0, \frac{1}{2}\right)$ determines $f$ on $[0,1)$. Repeating, on intervals $[n / 2,(n+1) / 2)$ for $n>0$, we find that

$$
f(x)=\frac{f\left(\frac{x}{2}\right)-\sum_{k=1}^{n} c_{k} f(x-k)}{c_{0}} \quad x \in[n, n+1) .
$$

We will call this relationship the forward substitution formula, as the value of $f$ in $[n, n+1)$ is given by evaluating $f$ in intervals strictly to the left of $n$.

As a first application of the forward substitution formula, observe that if $f$ is zero (almost) everywhere in $[0, \epsilon)$ then $f$ will be zero (almost) everywhere in $\mathbb{R}$. This means that we have aligned the left hand end of both $f$ 's support and the dilation equation's coefficients with 0 .

\section{Functions constant on integer intervals}

Figure 1 suggests an easy scheme for calculation, if we make the simplifying assumption that $f$ is constant on $[n, n+1)$ for all $n \in \mathbb{Z}$. Suppose we want to find out what dilation equation $\chi_{[0,2)}$ satisfies. 
Write out the values of our function on halfintervals we get:

Looking at the first half interval we see that $c_{0}=$ 1 , so we can fill row above.

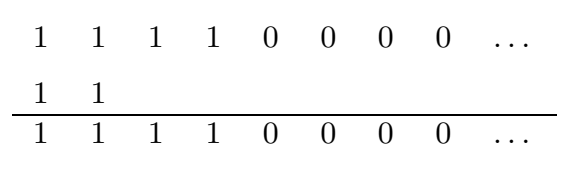

The next row is shifted along by one, and multiplied by $c_{1}$. The upper columns must sum to the bottom one, so by adding up column 2 we see $c_{1}=0$.

Now we repeat this process to fill in the next lines; first we need a 1 and then a 0 .

\begin{tabular}{ccccccccc} 
& 0 & 0 & & & & & & \\
1 & 1 & & & & & & & \\
\hline 1 & 1 & 1 & 1 & 0 & 0 & 0 & 0 & $\cdots$ \\
& & & & & & & & \\
& & & 0 & 0 & & & & \\
& & 1 & 1 & & & & & \\
& 0 & 0 & & & & & & \\
1 & 1 & & & & & & & \\
\hline 1 & 1 & 1 & 1 & 0 & 0 & 0 & 0 & $\ldots$
\end{tabular}

After this we can see that zeros will make all the columns add up. Reading the coefficients of the dilation off, we get $\chi_{[0,2)}$ satisfies $f(x)=1 f(2 x)+0 f(2 x-1)+1 f(2 x-2)$.

This process can also find out if there is a function which satisfies a dilation equation with given coefficients. For example the coefficients $1,1,-1,-1,1,1$ lead to the set $[0,1) \cup[2,3) \cup[4,5)$. This process should seem familiar. It is, of course, long division or polynomial division. Theorem 3.1 formalises this and is easy to verify.

Theorem 3.1. Consider the map from functions which are constant on $[n, n+1)$ to the polynomials given by:

$$
f(x)=\sum_{r} a_{r} \chi_{[r, r+1)}(x) \mapsto \sum_{r} a_{r} x^{r}=P_{f}(x) .
$$

Then this map is a linear bijection. When applied to a dilation equation it yields

$$
f(x)=\sum_{k} c_{k} f(m x-k) \Leftrightarrow \frac{x^{m}-1}{x-1} P\left(x^{m}\right)=Q(x) P_{f}(x),
$$

where $Q(x)=\sum c_{k} x^{k}$

Again, this is something with which we can do calculations. For example, look at the points where $x^{m}=x$. At $x=0$, we find that either $P(0)=0$ or $Q(0)=1$. This means that either $f(0)=0$ (which can be avoided by translation) or $c_{0}=1$. At $x=1$ we find that $P(1)=0$ or $Q(1)=m$, meaning that $f$ had mean zero, or the sum of the coefficients of the dilation equation was the scale.

We turn to the 2-refinable functions constant on $[n, n+1)$. These correspond to $P(x)$ where $P(x) Q(x)=P\left(x^{2}\right)(x+1)$ for some polynomial $Q$, so $P(x) \mid P\left(x^{2}\right)(x+1)$. It is quite easy to show that

$$
\left\{P(x): P(x) \mid P\left(x^{2}\right)(x+1)\right\}=\left\{R(x) /(x-1): R(x) \mid R\left(x^{2}\right), R(1)=0\right\},
$$

and $R(x)$ is relatively easy to pin-down, as the following Lemma shows.

Lemma 3.2. Let $R$ be a polynomial; then $R(x) \mid R\left(x^{2}\right)$ iff whenever $r$ is an order-p root of $R(x)$ then $r^{2}$ is a root of $R(x)$ of order at least $p$. Moreover, each root $r$ of $R(x)$ must have norm 0 or 1 and if $|r|=1$ then $r$ is a root of unity.

We now have a procedure for calculating possible $R(x)$. Draw a directed graph where the vertices are the roots of unity. A directed edge goes from one vertex to another if the second is the first squared. Next, choose a vertex on the graph and walk through the graph writing an increasing sequence of integers, one at each vertex. This walk ends up in a loop, at which stage we make all the integers in the loop the same. Now, we may stop or pick another root of unity and walk again. This provides the roots of $R(x)$, and the largest integer written by each vertex is 


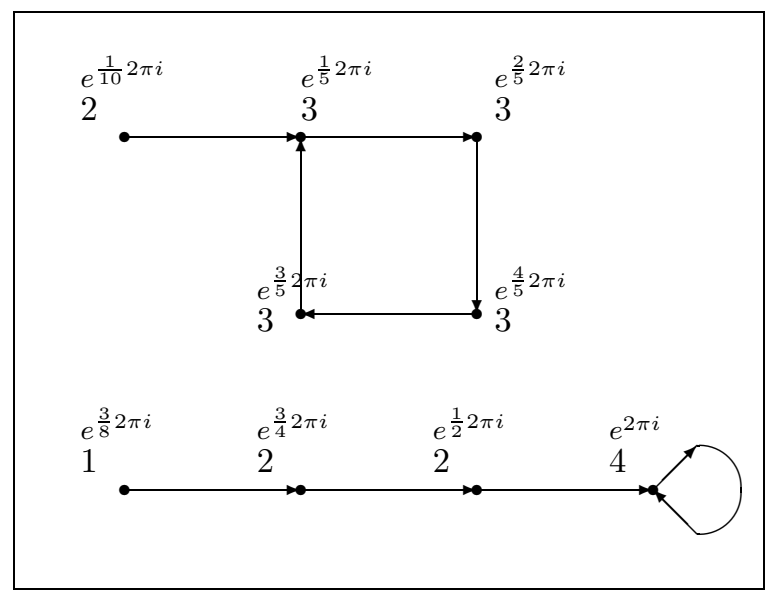

For this graph $\mathrm{R}(\mathrm{x})$ is

$$
\begin{aligned}
& {\left[\left(x-e^{\frac{1}{10} 2 \pi i}\right)\right]^{2}} \\
& \quad\left[\left(x-e^{\frac{1}{5} 2 \pi i}\right)\left(x-e^{\frac{2}{5} 2 \pi i}\right)\right. \\
& \left.\quad\left(x-e^{\frac{4}{5} 2 \pi i}\right)\left(x-e^{\frac{3}{5} 2 \pi i}\right)\right]^{3} \\
& {\left[\left(x-e^{\frac{3}{8} 2 \pi i}\right)\right][(x+i)(x+1)]^{2}} \\
& {[(x-1)]^{4} .}
\end{aligned}
$$

We have already visited 1 , so $P(x)=$ $R(x) /(x-1)$.

Figure 2: How to generate $P(x)$.

the order of the root. The roots of $P(x)$ are produced by beginning at 1 , if 1 has not already been visited, and finally decrementing the number written at 1 . Figure 2 illustrates this procedure.

Note that $P$ 's roots are a subset of $R$ 's so Lemma 3.2 tells us that $P$ 's roots are 0 or roots of unity. We can prevent 0 being a root of $P$ using translation (as in Lemma 2.1). Then we can prove the following result by showing the roots of $P$ are the same as the roots of $P(1 / x) x^{\operatorname{deg}(P)}$.

Theorem 3.3. If the coefficients of a polynomial $P$ are real, and $P$ 's roots have norm 1 , then $P$ is palindromic or anti-palindromic.

This means that real-valued refinable functions which are constant on $[n, n+1)$ are either symmetric or antisymmetric.

\section{Refinable characteristic functions}

In this section we are interested in searching for refinable characteristic functions on $\mathbb{R}$. The obvious example here is $\chi_{[0,1)}$ which is $m$-refinable for all $m \in \mathbb{N}^{+}$. Are there more exotic examples of refinable characteristic functions?

For scale $m>2$ the answer is yes. One example is provided by Cantor's middle third set, which satisfies $\chi_{S}(x)=\chi_{S}(3 x)+\chi_{S}(3 x-2)$. As a set of measure zero, this example might be dismissed. The study of self-affine tiles comes to the rescue here. In Section V.B of [3] it is shown there is a tile whose characteristic function satisfies the dilation equation $f(x)=f(3 x)+f(3 x-2)+f(3 x-4)$, which is definitely not a simple union of intervals.

However, the only self-affine tiles of scale 2 are simple intervals. Also, there are refinable characteristic functions, such as those exhibited at the start of Section 3, which do not fulfill the requirements to be self-affine tiles. Looking at this problem using Figure 1 gives us the following result.

Theorem 4.1. If $S \subset \mathbb{R}$ is bounded with positive measure and satisfies a dilation equation $\chi_{S}(x)=$ $\sum c_{k} \chi_{S}(2 x-k)$ a.e., where $c_{0} \neq 0$ and $c_{k}=0$ when $k<0$, then $c_{0}=1$, the rest of the $c_{k}$ are integers with $\left|c_{k}\right| \leq 2^{k}$ and $S$ has positive measure in both $\left[0, \frac{1}{2}\right)$ and $\left[\frac{1}{2}, 1\right)$.

More information than this seems difficult to extract, but we are presented with a way to search for examples. Let $S_{n}=S \cap[n, n+1)$. Examine the half interval in Figure 1 starting at $n / 2$. At the bottom of the picture we have the half of the set $S_{\lfloor n / 2\rfloor}$. Above this we have $\frac{S_{0}+n}{2}, \frac{S_{1}+n-1}{2}, \frac{S_{2}+n-2}{2}, \ldots$ and from half-interval to half-interval the alignment of these sets does not change. Now consider the maps $V_{n}:[n / 2,(n+1) / 2) \rightarrow\{0,1\}^{n+1}$ defined by

$$
V_{n}=\chi_{\frac{s_{0}+n}{2}} \times \chi_{\frac{s_{1}+n-1}{2}} \times \chi_{\frac{s_{2}+n-2}{2}} \times \ldots
$$


Note that the first $n+1$ components of $V_{n+1}(x)$ agree with the components of $V_{n}\left(x-\frac{1}{2}\right)$. We are interested in the values which these $V_{n}$ functions take. To allow almost-everywhere solutions, we consider the list of values $L_{n}$ which $V_{n}$ achieves on sets of positive measure. Given $L_{n}$ and $c_{0}, \ldots, c_{n}$ we are going to determine all possible combinations of $c_{n+1}$ and $L_{n+1}$.

By Theorem 4.1, we know that $c_{0}=1$ and $\chi_{S_{0}}$ takes the value 1 for at least one combination in $L_{n}$. Using the forward substitution formula we rearrange for $c_{n+1}$

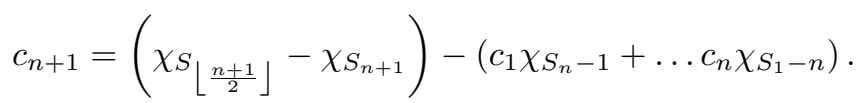

We may have to consider $-1,0$ or 1 for the value of the first bracket and the values in the second bracket are calculated from $L_{n}$.

We now have set of candidate values for $c_{n+1}$. For each of the $c_{n+1}$ we then produce a set of possible values $L_{n+1}$ by checking what values $\chi_{S_{n+1}}$ would take. For each old possibility $l \in L_{n}$ and we may be able to add new possibilities $(l 0),(l 1)$ or $(l 0, l 1)$ to $L_{n+1}$ in accordance with what is allowed by the forward substitution formula.

Thus we have produced a list of values $c_{n+1}$ with corresponding possibilities for $L_{n+1}$ and have moved to the next stage of the recursive search. This process branches rapidly; we may have as many as 3 choices for $c_{n+1}$ and $3^{\# L_{n}}$ choices for $L_{n+1}$. This makes the search impractical by hand, but possible by computer.

After running a search for sequences of up to 6 coefficients, a list of about 30 possible sets coefficients was obtained. Many of these could be rejected as they would have no $L^{1}$ solution [4]. The remainder could not have exotic characteristic functions as solutions because $L^{1}$ solutions are known to be unique and other solutions could be exhibited using Section 3.

This method is quite crude, in that it completely ignores geometry and considers only arithmetic. It produces suggestions which we already know are unions of integer intervals so we know it lacks important information about the problem. Nonetheless, its overeager suggestions have failed to find any counterexamples and we conjecture that there are no 2-refinable exotic characteristic functions.

\section{Smoothness and boundedness}

If $f$ is continuous on $(0,1)$, then it will be continuous on $(1,2)$ as the forward substitution formula tells us that $f(x)=\left(f(x / 2)-c_{1} f(x-1)\right) / c_{0}$. If $f$ is continuous at 0 , then $f(0)=0$, because we have translated $f$ so that $f(x)=0$ when $x<0$. Now, $f$ continuous on $[0,1)$ gives $f$ continuous on $[1,2)$ for a combination of two reasons:

- the term $f(x-1)$ grows continuously from 0 at 1 as $f(x)$ grows continuously from 0 at 0 , so the sum on $[0,1)$ makes a continuous transition to the sum on $[1,2)$,

- the remaining terms (in this case just $f(x / 2)$ ) are continuous where they are being evaluated (that is to the left of 1 where we know $f$ is continuous).

By induction, the continuity of $f$ on $[0,1)$ is necessary and sufficient for the continuity of $f$ as a whole. Similar arguments can be applied to differentiability and other sorts of smoothness.

On $[0,1 / 2)$ the forward substitution formula has the simple form $f(x)=c_{0} f(2 x)$. It would seem profitable to determine the implications of this combined on smoothness. By iterating toward the origin we get the following result.

Lemma 5.1. Suppose we are given compactly-supported $f$ which satisfies a dilation equation with $c_{k}=0$ when $k<0$. Then if $f \in C^{r}$ we have $\left|c_{0}\right|<2^{-r}$ or $f$ is zero.

Corollary 5.2. There are no non-zero compactly-supported $C^{\infty}$ solutions to finite dilation equations. 


\begin{tabular}{r|rr|rrr|rrr} 
& \multicolumn{2}{|c|}{ Coefficients } & \multicolumn{3}{c|}{ TLoW } & \multicolumn{3}{c}{ SRCfSS } \\
$\mathrm{N}$ & $\left|c_{0}\right|$ & $-\log _{2}\left|c_{0}\right|$ & $\mathrm{p} 226$ & $\mathrm{p} 232$ & $\mathrm{p} 239$ & $r_{20}$ & $r_{\infty}$ & $\mathrm{UB}$ \\
\hline 2 & 0.683 & 0.550 & 0.339 & 0.500 & 0.550 & 0.550 & 0.550 & 0.550 \\
3 & 0.470 & 1.0878 & 0.636 & 0.915 & 1.0878 & 1.0831 & 1.0878 & 1.0878 \\
4 & 0.325 & 1.6179 & 0.913 & 1.275 & 1.6179 & 1.6066 & 1.6179 & 1.6179 \\
5 & 0.2264 & 2.1429 & 1.177 & 1.596 & & 1.9424 & & 1.9689 \\
6 & 0.1577 & 2.6644 & 1.432 & 1.888 & & 2.1637 & & 2.1891 \\
7 & 0.1109 & 3.1831 & 1.682 & 2.158 & & 2.4348 & & 2.4604
\end{tabular}

Various estimates of $r+s$ for Daubechies's family of extremal phase wavelets (see [2] page 195). The upper bounds derived in Corollary 5.3 are shown as $-\log _{2}\left|c_{0}\right|$. The values in the TLoW column are lower bounds taken from tables in [2]. The column labeled SRCfSS is taken from [5] (page 1570) and shows lower bounds $\left(r_{20}\right.$ and $\left.r_{\infty}\right)$ and an upper bound.

Figure 3: Estimates of smoothness for Daubechies's extremal phase wavelets

Note that this result does not extend to the non-compactly-supported case; $f(x)=x$ is a noncompactly-supported analytic function satisfying $f(x)=1 / 4 f(2 x+1)+1 / 4 f(2 x-1)$. However, Lemma 5.1 extends nicely to $C^{r+s}$ where $r \in \mathbb{N}$ and $0<s<1$ is the Hölder exponent of continuity of the $r^{\text {th }}$ derivative.

Corollary 5.3. Suppose we are given compactly-supported $f$ which satisfies a dilation equation with $c_{k}=0$ when $k<0$. Then if $f \in C^{r+s}$ we have $\left|c_{0}\right| \leq 2^{-(r+s)}$ or $f$ is zero.

Figure 3 shows that this bound on smoothness can be surprisingly good.

These results can also be extended to give an estimate of the growth rate of a refinable function. Compare this to the bound given in Theorem 4.1.

Theorem 5.4. Suppose a compactly-supported $f$ satisfies a dilation equation with $c_{k}=0$ when $k<0$, then if $f$ is bounded on $[0,1)$ we will find $f$ is bounded by $C(M+1)^{n}$ on $[n, n+1)$ where $M=\max _{k>1}\left|c_{k} / c_{0}\right|$.

\section{2- and 3-refinable functions}

When we search for functions which simultaneously satisfy two dilation equations of unrelated scales, examples seem thin on the ground. The obvious examples all seem to arise from $\chi_{[0,1)}$ via differentiation, integration and convolution.

Our basic trick is to use the simple form of the forward substitution formula on the first interval: $f(x)=c_{0} f(2 x)=d_{0} f(3 x)$. This turns out to be a strong condition. By combining this with integrability we can determine $f$.

Theorem 6.1. Suppose $f$ is 2- and 3-refinable, say:

$$
f(x)=\sum_{k} c_{k} f(2 x-k)=\sum_{k} d_{k} f(3 x-k),
$$

and $c_{0} \neq 0$ and $c_{k}=0$ when $k<0$. Suppose further that $f$ is integrable on some interval $[0, \epsilon]$, then $f(x)=\gamma x^{\beta}$ on $[0,1)$ where $\beta=-\log _{2} c_{0}=-\log _{3} d_{0}$.

Proof. By iterating the forward substitution formula we find for $k, l \in \mathbb{Z}$

$$
f(x)=c_{0}^{k} d_{0}^{l} f\left(2^{k} 3^{l} x\right),
$$

as long as both sides are evaluated in $[0,1 / 3)$. Set $F(x):=\int_{0}^{x} f(t) d t$ for $x \in[0, \epsilon)$, then $F$ is continuous and $F^{\prime}(x)=f(x)$ for almost every $x \in[0, \epsilon)$.

Choose $\alpha \in[0, \epsilon)$ so that $F(\alpha) \neq 0$. Using the continuity of $F$ and performing a change of variable using Equation 3 we can show that $\log _{2} c_{0}=\log _{3} d_{0}=-\beta$ and $F\left(2^{k} 3^{l} \alpha\right)=\left(2^{k} 3^{l}\right)^{\beta+1} F(\alpha)$. 
This $F$ agrees with $x^{\beta+1} \frac{F(\alpha)}{\alpha^{\beta+1}}$ on the dense subset $\left\{2^{k} 3^{l} \alpha\right\} \cap[0, \epsilon)$. Both are continuous so they must be the same and

$$
f(x)=F^{\prime}(x)=(\beta+1) x^{\beta} \frac{F(\alpha)}{\alpha^{\beta+1}} .
$$

Finally, extend $f$ to all of $[0,1)$ using $f(x)=d_{0} f(3 x)$.

Once we have $f$ on $[0,1)$ the forward chaining formula can do the rest of the work for us. Solutions to two such dilation equations must be of the form:

$$
f(x)=\sum_{l} a_{l}(x-l)_{+}^{\beta},
$$

where $x_{+}$is $x$ if $x>0$ and zero otherwise. If this sum is eventually to cancel to make $f$ compactly supported, then $\beta \in \mathbb{N}$. Indeed, once $\beta \in \mathbb{N}$ then a simple linear constraints on the $a_{l}$ are a necessary and sufficient condition for compact support.

We can extend this result into $L^{1} \cup L^{2}$, providing that at least one compactly-supported solution exists. In this case the $L^{1} \cup L^{2}$ solution space of the pair of scale 2 and scale 3 equations is spanned by the compactly-supported solution $f$ and its Hilbert transform $\mathcal{H} f$.

When no compactly-supported solution exists our technique is of no help. One can exhibit $L^{p}$ functions such as $f(x)=|x|^{\beta}-|x-1|^{\beta}$ which are 2- and 3-refinable via the equations $f(x)=$ $2^{-\beta}(f(2 x)+f(2 x-1))$ and $f(x)=3^{-\beta}(f(3 x)+f(3 x-1)+f(3 x-2))$. The standard analysis of dilation equations shows that these dilation equations have no compactly-supported $L^{p}$ solutions for most values of $\beta$.

\section{Parallelepipeds as self-affine tiles}

A self-affine tile $G \subset \mathbb{R}^{d}$ has essentially disjoint integer translates which cover $\mathbb{R}^{d}$ and satisfies $A G=\bigcup\left(G+\vec{k}_{i}\right)$ for a dilation ${ }^{1}$ matrix $A$ and integer vectors $\vec{k}_{i}$. Self-affine tiles are of interest as they can be used to build simple orthogonal bases.

Self-affine tiles come in many shapes including from parallelepipeds to twin-dragons and Sierpinski gaskets. For a fixed dilation matrix $A$, tiles of different shapes may be possible. Here we show when $A$ admits a parallelepipeds as a self-affine tile. We begin with the equivalent of our line-up lemmas.

Lemma 7.1. Suppose we have a matrix $A$ and a parallelepiped $P$ such that:

$$
A P=\left(P+\vec{k}_{0}\right) \cup\left(P+\vec{k}_{1}\right) \cup \ldots \cup\left(P+\vec{k}_{q-1}\right)
$$

is an essentially disjoint union, then each corner of $A P$ is in exactly one of $P+\vec{k}_{0}, P+\vec{k}_{1}, \ldots, P+$ $\vec{k}_{q-1}$.

Now we have identified the corners of $A P$ we concentrate on what happens to the edges at a given corner. This provides information about $A$.

Theorem 7.2. Suppose we have a matrix $A$ and a parallelepiped $P$ as in Lemma 7.1, then $A$ is similar to a weighted permutation matrix where the weights are integers.

Proof. Pick any corner $\vec{c}$ of $A P$, then $\vec{c}=A \vec{b}$ for some corner $\vec{b}$ of $P$. Let the edges of $P$ at $\vec{b}$ be $\vec{b}+\vec{e}_{1}, \ldots, \vec{b}+\vec{e}_{d}$. By applying $A$ we find the edges at $\vec{c}$ are $\vec{c}+A \vec{e}_{1}, \ldots, \vec{c}+A \vec{e}_{d}$.

Using Lemma 7.1 we can also write this corner as $\vec{c}=\vec{d}+\vec{k}_{r}$. The edges of $P$ at $\vec{d}$ must be the same as those at $\vec{b}$, though possibly pointing in the opposite direction, so they are $\vec{d}+s_{1} \vec{e}_{1}, \ldots, \vec{d}+$ $s_{d} \vec{e}_{d}$ where $s_{i}= \pm 1$. Thus the edges of $P+\vec{k}_{r} \subset A P$ are $\vec{c}+s_{1} \vec{e}_{1}, \ldots, \vec{c}+s_{d} \vec{e}_{d}$. As $P+\vec{k}_{r}$ is the only part of the union forming $A P$ at $\vec{c}$, these edges must be parallel, but may be shorter than the edges $\vec{c}+A \vec{e}_{1}, \ldots, \vec{c}+A \vec{e}_{d}$. So $\vec{c}+A \vec{e}_{i}=\vec{c}+\alpha_{i} \vec{e}_{j}$ where $\left|\alpha_{j}\right| \geq 1$.

\footnotetext{
${ }^{1} \mathrm{~A}$ dilation matrix is one whose eigenvalues have norm bigger than 1 .
} 
Consider how the translates of $P$ pack into $A P$. We can count how many copies of $P$ are along each edge. Hence, the side of $A P$ parallel to $\vec{e}_{j}$ must be a whole multiple of $\vec{e}_{j}$. We conclude that $A \vec{e}_{i}=\alpha_{i} \vec{e}_{j},\left|\alpha_{j}\right| \in \mathbb{N}^{+}$and so $A$ permutes the edges with integer weights. As the edges form a basis for $\mathbb{R}^{d}, A$ must be weighted permutation matrix.

By raising the $A$ to the order of the permutation we get the following.

Corollary 7.3. With $A$ as in Lemma 7.1 we have $A^{l}$ is similar to a diagonal matrix (via a real matrix) for some $l$ dividing $\operatorname{lcm}(1,2, \ldots, d)$. Consequently $A$ is diagonalisable.

By considering how we can pack a prime number of parallelepipeds into a larger parallelepiped we can prove Corollary 7.4. We assume the matrix is expanding to force the permutation to be a cycle of length $d$. This is the tip of the iceberg of the relationship between number theory and self-affine tiles [6].

Corollary 7.4. If $A$ is an expanding matrix with $\operatorname{det}(A)$ prime and $A$ has a tile as in Lemma 7.1, then $A^{d}=\beta I$.

If we allow the $\vec{k}_{i}$ to have non-integer values then $A$ being similar to a weighted permutation matrix, where the weights are non-zero integers is a necessary and sufficient for the existence of parallelepiped tile.

Theorem 7.5. Suppose $A$ is similar to a weighted permutation matrix, where the weights are non-zero integers, then there exists a parallelepiped $P$ and vectors $\vec{k}_{i}$ such that:

$$
A P=\left(P+\vec{k}_{0}\right) \cup\left(P+\vec{k}_{1}\right) \cup \ldots \cup\left(P+\vec{k}_{q-1}\right) .
$$

\section{References}

[1] Xinrong Dai, Qiyu Sun, and Zeyin Zhang, Compactly supported both $m$ and $n$ refinable distributions II, East Journal of Approximations 6 (2000), 201-209.

[2] Ingrid Daubechies, Ten lectures on wavelets, CBMS, vol. 61, SIAM, Philadelphia, 1992.

[3] K. Gröchenig and W. R. Madych, Multiresolution analysis, Haar bases, and self-similar tilings of $\mathbb{R}^{n}$, IEEE Transactions on Information Theory 38 (1992), no. 2, 556-568.

[4] Christopher Heil and David Colella, Sobolev regularity for refinement equations via ergodic theory, Wavelets and Multilevel Approximation (C. K. Chui and L. L. Schumaker, eds.), Approximation Theory VIII, vol. 2, World Scientific Publishing, Singapore, 1995, pp. 151-158.

[5] Oliver Rioul, Sample regularity criteria for subdivision schemes, SIAM Journal of Mathematical Analysis 23 (1992), no. 6, 1544-1576.

[6] Yang Wang, Self-affine tiles, Advances in Mathematics 121 (1996), no. 1, 21-49. 